Western University

Scholarship@Western

$4-1-2013$

\title{
A magnetic-resonance-imaging-compatible remote catheter navigation system.
}

Mohammad Ali Tavallaei

Yogesh Thakur

Syed Haider

Maria Drangova

Follow this and additional works at: https://ir.lib.uwo.ca/biophysicspub

Part of the Medical Biophysics Commons

\section{Citation of this paper:}

Tavallaei, Mohammad Ali; Thakur, Yogesh; Haider, Syed; and Drangova, Maria, "A magnetic-resonanceimaging-compatible remote catheter navigation system." (2013). Medical Biophysics Publications. 87. https://ir.lib.uwo.ca/biophysicspub/87 


\title{
A Magnetic Resonance Imaging Compatible Remote Catheter Navigation System
}

\author{
Mohammad Ali Tavallaei*, Yogesh Thakur, Syed Haider, and Maria Drangova
}

\begin{abstract}
A remote catheter navigation system compatible with magnetic resonance imaging has been developed to facilitate magnetic resonance image guided catheterization procedures. The interventionalist's conventional motions (axial motion and rotation) on an input catheter - acting as the master - are measured by a pair of optical encoders and a custom embedded system relays the motions to a pair of ultrasonic motors. The ultrasonic motors drive the patient catheter (slave) within the MRI scanner, replicating the motion of the input catheter. The performance of the remote catheter navigation system was evaluated in terms of accuracy and delay of motion replication outside and within the bore of the magnet. While inside the scanner bore, motion accuracy was characterized during the acquisition of frequently used imaging sequences, including realtime GRE acquisition. The effect of the catheter navigation system on image SNR was also evaluated. The results show that the master-slave system has a maximum time delay of $41 \pm 21 \mathrm{~ms}$ in replicating motion; an absolute value error of $2 \pm 2^{\circ}$ was measured for radial catheter motion replication over $360^{\circ}$ and 1.1 $\pm 0.8 \mathrm{~mm}$ in axial catheter motion replication over $100 \mathrm{~mm}$ of travel. The worst case SNR drop (in spin echo images) was observed to be $2.5 \%$.
\end{abstract}

Index Terms-Catheterization, image guided interventions, magnetic resonance imaging, master-slave, piezoelectric actuators, real-time systems, remote navigation, surgical robotics, telerobotics.

\section{INTRODUCTION}

$\mathrm{C}$ ARDIAC catheterization has become an essential tool in the management of cardiac and vascular diseases, in general, and the treatment of cardiac arrhythmias, in particular. The conventional approach to percutaneous

"M.A. Tavallaei is with the Graduate Program in Biomedical Engineering and the Robarts Research Institute, The University of Western Ontario, London, ON, N6A 5B9, Canada (e-mail: atavall@robarts.ca).

Y. Thakur is a clinical instructor in the Faculty of Medicine - University of British Columbia and a medical physicist at the Vancouver Coastal Health Authority, Vancouver, BC, V5Z1M9, Canada(e-mail: yogesh.thakur@vch.ca)

Syed Haider was a technical specialist at the Robarts Research Institute, London, ON, N6A 5K8. He is currently with Sunnybrook Hospital, Toronto, ON, M4N3M5, Canada (e-mail: syed.haider@sri.utoronto.ca )

M. Drangova is with the Department of Medical Biophysics, Schulich School of Medicine \& Dentistry, The University of Western Ontario, London ON N6A5K8, Canada. She is also with the Graduate Program in Biomedical Engineering and the Robarts Research Institute, The University of Western Ontario, London, ON N6A 5B9, Canada, She is also with the Heart and Stroke Foundation of Ontario, Toronto, ON, M4P 1E4, Canada (e-mail: mdrangova@robarts.ca ) transluminal catheter procedures relies on fluoroscopic $\mathrm{x}$-ray imaging as the main modality for guiding the interventionalist during the procedure. Fluoroscopically guided catheterization provides two-dimensional (2D) projections of the anatomical site in real time and is limited by low tissue contrast, making interpretation of the complex three-dimensional (3D) anatomy difficult. Furthermore, fluoroscopy-guided catheterization exposes both patients and staff $[1,2]$ to radiation. Interventionalists and staff must wear heavy lead aprons during the long procedures, commonly resulting in physical strain[3]. Considering the large number of cardiac catheterizations $(1,059,000$ in USA) and percutaneous coronary interventions (622,000 in USA) performed annually [4] and the general upward trend of the number of catheterization procedures, numerous avenues of improving the procedures - in terms of improved efficacy and ease of delivery - are being explored.

Significant developments aimed at reducing exposure of the interventionalist and staff while maintaining procedure efficacy, have included a series of robotically guided catheter manipulators [5-9] or magnetically guided navigation systems $[10,11]$. For electrophysiological procedures, 3D electrical mapping, using systems such as Carto (Biosense Webster) or EnSite NavX (St. Jude Medical), has provided the ability to visualize the catheter in relation to a 3D electrical map. However, guidance is still subject to low anatomical contrast, and the inability to visualize soft tissues and lesions limits the efficacy of the treatment.

Magnetic Resonance Imaging (MRI) allows for high contrast visualization of soft tissue in $3 \mathrm{D}$ and has been shown to differentiate between ischemic, infarcted, and arrhythmogenic tissue in the heart [12]. These advantages of MRI make it an attractive modality for guiding catheter-based treatments. Recent developments have demonstrated the ability to acquire MR images at high frame rates [13-15], demonstrating the potential for MRI to become a source of image feedback for image guided minimally invasive interventions, particularly of percutaneous transluminal catheter procedures [16]. MRI-guidance of catheterization has been demonstrated in animals as early as the late 1990's [17] and Razavi et al. [18] used MRI-guided cardiac catheterization on humans in 2003. Although these MRI methods show promise [19], practical implementation requires modifications to most equipment peripheral to the image acquisition (e.g. magnetically shielded monitors, controls within the scanner room and specialized noise suppressing headsets to permit communication during the procedure). Another important constraint imposed during an MRI guided procedure is the 
requirement for the interventionalist to reach within the MRI scanner bore in order to reach the catheter manipulation site on the patient. The catheterization can be specially challenging with unfavorable entry sites and angles. Although open bore and wide bore scanners may partially alleviate some of these problems, their numbers are limited and it is unlikely that clear access to the patient will be possible in the foreseeable future. Therefore the mentioned problems continue to limit the transition from fluoroscopic-guided to MRI-guided catheterization.

The developments presented in this paper aim to facilitate MRI guided catheterization by allowing the interventionalist to perform the catheter navigation from a location remote to the MRI scanner. Specifically, a Magnetic Resonance compatible Remote Catheter Navigation System (MR-RCNS) was designed and built. The MR-RCNS allows the interventionalist to apply conventional push/pull and rotate motions on an input catheter and have the motions replicated on a remote patient catheter by an MR compatible slave robot inside the magnet room, thereby freeing the interventionalist from the workspace constraints of the MRI scanner.

This paper is organized as follows: first the mechatronics design of the master-slave system is described in section II, the methods of evaluating the system's performance and its electromagnetic interference are detailed in section III and the results of evaluation are given in section IV. Finally a discussion and conclusions are presented in sections V and VI, respectively.

\section{SYSTEM DESCRIPTION}

The MR-RCNS is designed as a master-slave system that takes advantage of an interventionalist's existing dexterous skills - relying on the push/pull and twist motions conventionally imparted on a catheter during manipulation. The design is based on prior developments of a remote catheter navigation system $[8,20]$ designed and evaluated by our group. Each component of the new MR-RCNS is described below.

\section{A. Catheter Sensor - master}

The sensor, which remains outside the magnet room, does not need to be MR compatible, so the original design was used [20] (note that, with minor modification, the sensor can be made MR-compatible and used within the scanner room if necessary). The role of the catheter sensor (CS) is to enable the measurement of the radial and axial motions imparted by the interventionalist on an input catheter. As described in detail in $[8,20,21]$, the CS utilizes a pair of optical encoders coupled to the input catheter. The angle measurements of the encoders are transmitted directly to the motor servomechanism (see below) and used to determine the motion of the catheter manipulator.

\section{B. Catheter Manipulator - slave}

The catheter manipulator (CM) must replicate the motion imparted on the input catheter and must operate within the environment of a clinical MRI scanner - where it is subjected to strong (up to $3 \mathrm{~T}$ ) and rapidly switching magnetic fields (gradients). Therefore, a redesign of the manipulator described in [8] was required, while the principle of operation was maintained (Fig. 1). The patient catheter is moved in the axial direction using a set of rollers that grip the catheter and



Fig. 1. The MR-RCNS is shown. The interventionalist applies conventional motion on the input catheter in the sensory system shown on the left and the MRI compatible manipulator shown on the right of the image replicates that motion on a patient catheter.

are driven by a motor mounted on a rotating gantry. The rotating gantry provides radial motion of the entire assembly, including the catheter. An added modification is that the bases of these rollers are mounted on a moving plane. Manual rotation of a knob connected to a screw moves the plane and allows adjustment of the position of the rollers, thereby accommodating various gauge catheters. Unscrewing the knob completely allows for easy insertion or withdrawal of the catheter by separating the opposing rollers. All gantry components are manufactured out of derlin and the urethane rollers are held using stainless-steel springs. A slip ring (AC6438, Moog Inc., East Aurora NY, USA) is used to transfer the electrical control signals to the axial motor, as the gantry is rotated, via a sprocket and non-magnetic chain drive.

In case of an emergency, the manipulator can be moved back manually and the catheter can be extracted from the point of entry or the catheter may be pulled on directly from the robot.

Studies of catheter dynamics in conventional catheter navigation [21] showed that the minimum needed force and torque requirements are $0.29 \pm 0.06 \mathrm{~N}$ and $1.15 \pm 0.3 \mathrm{mNm}$ respectively. Also the peak velocities were measured to be $360 \pm 180 \mathrm{~mm} . \mathrm{s}^{-1}$ and $19 \pm 7 \mathrm{rad}_{\mathrm{s}}{ }^{-1}$ for axial and radial catheter motion. To satisfy these requirements a pair of ultrasonic motors made of non-ferromagnetic material (USM45 and USM60, Xi'an Ultrasonic Technology Co., LTD., China) with a maximum torque of $0.4 \mathrm{Nm}$, nominal torque of $\sim 0.1 \mathrm{Nm}$ and peak speed of $320 \mathrm{rpm}$ were used for actuation. Ultrasonic motors were selected because they produce no backlash, have a hard stop even without power and allow precise positioning [22]. For axial motion, force is applied on the catheter through rollers (radius $\sim 3 \mathrm{~mm}$ ). Therefore with sufficient friction 
between the rollers and catheter the nominal torque of the motors can easily provide forces exceeding the peak requirement in catheterization $(0.3 \mathrm{~N})$.

\section{Ultrasonic Motor Servomechanism}

The CM of the MR-RCNS must be able to replicate the motions measured by the CS in near real-time. To achieve this fast response an embedded system was designed and built to control the motors of the CM. The embedded system simultaneously measures the encoder positions of corresponding joints of the master and slave and compares the two values to calculate an error. A control signal proportional to this error is calculated by the embedded system and applied to the ultrasonic motor driver. This implementation differs from the original RCNS [8], as it no longer requires a dedicated workstation.

The embedded system uses an 8-bit microcontroller (ATMEL Inc., San Jose, California USA) with a clock cycle rate of $8 \mathrm{MHz}$ that results in a closed loop control rate of approximately $3 \mathrm{kHz}$, as implemented. The encoder position of each joint can be logged to a personal computer at a sampling rate 100 times slower than the control rate $(30 \mathrm{~Hz})$ through a serial RS-232 port. The microcontroller uses interrupt service routines to perform tasks such as serial communication or measuring the encoder positions.

Separate servomechanisms - comprising a sensor encoder, an embedded system, and an ultrasonic motor - were assembled for the axial and radial motions. To minimize electromagnetic interference from the servomechanism, all wires were shielded and the shields were grounded.

\section{EvaluATION}

\section{A. Evaluation of the Servomechanism}

\section{Accuracy and Robustness}

To evaluate the accuracy of the servomechanism and its robustness to increased loading, the step response of the servomechanism was studied. Weights (up to $500 \mathrm{~g}$ ) were suspended from a pulley of radius $2.25 \mathrm{~cm}$ mounted to the shaft of the motor. This provided torques of $0.11 \mathrm{Nm}$ that is close to the motor's nominal torque $(0.1 \mathrm{Nm})$. The response of the servomechanism was recorded following a $90^{\circ}$ input angle. For each load (torque ranges of $0-0.11 \mathrm{Nm}$ ), the step response was measured 20 times.

\section{Dynamic Motion Replication}

To validate the dynamic motion replication capabilities of the servomechanism manual motion profiles were applied to an encoder wheel, acting as a master joint. The encoder positions of this master joint and the motor were logged to a personal computer through the embedded system. Each manual motion profile consisted of 40 revolutions in the clockwise and anticlockwise directions; twenty sets of motion profiles were evaluated. These experiments were performed under the maximum loading conditions $-0.11 \mathrm{Nm}$. The delay in motion replication was determined by cross-correlating the input and replicated motion profiles using MATLAB (MathWorks Inc., Massachusetts USA).

\section{B. Evaluation of the MR-RCNS}

Following the initial evaluation of the servomechanisms, the accuracy and precision of the entire MR-RCNS was evaluated inside the bore of a clinical MRI scanner (3 T, Discovery 750, software revision 22M32, General Electric Healthcare, USA). For the imaging experiments the 32channel cardiac transmit-receive radiofrequency (RF) coil was used. The CS and the embedded systems were placed in the scanner's control room and the wire connections for the motor drive and encoder signals were passed through 1,000 $\mathrm{pF}$ RF filters with a $3 \mathrm{~dB}$ cut-off frequency of $3.2 \mathrm{MHz}$. These filters were required to minimize the introduction of external RF noise into the MR scanner suite and RF interference with motor controllers and embedded system during image acquisition.

The MR-RCNS slave was placed on the patient bed within the scanner bore at a distance of approximately $60 \mathrm{~cm}$ from the magnet isocentre. Ablation catheters (6F-7F, Biosense Webster Inc.) were used for both the input and patient catheters; these were confined to travel within 6-mm diameter Plexiglas tubes for all experiments.

The accuracy tests were performed during an imaging session to evaluate any effects image acquisition may have on the manipulator performance. For these experiments, the effect of two pulse sequences used in cardiac imaging were evaluated: FIESTA - a steady state free precession pulse sequence - (FOV $24 \mathrm{~cm}$, slice thickness $6 \mathrm{~mm}$, TR $4.5 \mathrm{~ms}$, TE $1.7 \mathrm{~ms}, \mathrm{FA} 45^{\circ}$, matrix 256x256, and BW $125 \mathrm{kHz}, \mathrm{NEX} 4$ ) and FGRET - a real-time multi-echo fast gradient echo pulse sequence - (FOV $24 \mathrm{~cm}$, slice thickness $10 \mathrm{~mm}$, TR $10.5 \mathrm{~ms}$, TE $1.4 \mathrm{~ms}$, FA $12^{\circ}$, matrix $128 \times 96$, and BW $125 \mathrm{kHz}$, echo train length 8 ). Each sequence was repeated continuously for the duration of the experiments and for the FGRET sequence the imaging plane was continuously altered to simulate a realtime catheter-guidance experiment.

\section{Axial Motion Accuracy}

To measure axial accuracy the input catheter was moved over a distance of $127 \mathrm{~mm}$ from a starting position; the experiment was repeated ten times in each direction. The position of the tip of the input catheter was measured using calipers and that of the patient catheter was marked on a ruler then measured using calipers; in each case care was taken to avoid parallax.

\section{Radial Motion Accuracy}

Radial accuracy was evaluated using protractors mounted at the distal end of each Plexiglas tube; a pair of pointers mounted on the catheters was used to indicate the radial position. The master was rotated 3,600 degrees in the clockwise (and anticlockwise) direction ten times; the angle of the input and output catheters was recorded at the end of each motion for each direction.

\section{Evaluation of the effects of the RCNS on MR images}

A concern when introducing electronic devices within an MRI scanner is that RF noise from the devices can potentially introduce noise and artifacts within the MR images. To determine any detrimental effects of the MR-RCNS on the 
MR images, we followed the guidelines for measuring signalto-noise ratio (SNR) outlined by the National Electrical Manufacturer's Association (NEMA) [23]. Specifically, a 17$\mathrm{cm}$ diameter water phantom (MRS HD sphere, model 2152220; General Electric, Milwaukee, WI, USA), doped with metabolite salts and gadolinium-based contrast agent [24] was used; the $T_{1}$ and $T_{2}$ values of the solution were $392 \mathrm{~ms}$ and $297 \mathrm{~ms}$, respectively. The Spin Echo (SE) pulse sequence was used (FOV $24 \mathrm{~cm}$, slice thickness $6 \mathrm{~mm}$, TR 1,300 ms, TE $20 \mathrm{~ms}$, matrix 256x256, and BW $15.6 \mathrm{kHz}$ ). All geometric corrections and filters were turned off for the experiments. All gain settings were maintained constant throughout the experiment. The room and phantom temperature were $19.5^{\circ} \mathrm{C}$.

The effect of the MR-RCNS on image SNR was evaluated with the CM positioned at approximately $70 \mathrm{~cm}$ and $40 \mathrm{~cm}$ from the isocentre; these positions were chosen as they represent the expected range of positions during actual catheterization procedures. For both sequences, images were acquired at each position at baseline and during each of the following three states: 1) RCNS connected to the servomechanism via the $1,000 \mathrm{pF}$ filters; 2) all RCNS electronics turned on but no motion applied; and 3) the input catheter (in the console room) was moved thereby actuating the RCNS motors (on the scanner bed).

Noise in the images was calculated using method 1 outlined in the NEMA protocol [23]. Specifically, two consecutively acquired images of the same slice were subtracted and the standard deviation $(\sigma)$ in an $11 \times 11$ pixel region of interest (ROI) in the center of the difference image was calculated; the noise in the region was then calculated as $\sigma / \sqrt{2}$ to correct for the difference operation. Image signal was calculated as the average intensity of a 7x7 pixel ROI in the center of the first image. The signal to noise ratio (SNR) was calculated from the central axial slice of the acquired images, for all four conditions mentioned above.

\section{RESUlTS}

\section{A. Evaluation of Servomechanism}

\section{Accuracy and Robustness}

Representative curves for the step response of the servomechanism system for a reference value of $90^{\circ}$ is shown in Fig. 2 for no load and a maximum load of $0.11 \mathrm{Nm}$. In all cases - multiple repetitions and different loading conditions no overshoot or offset was observed, at the $30 \mathrm{~Hz}$ sampling rate used to record the angular position.
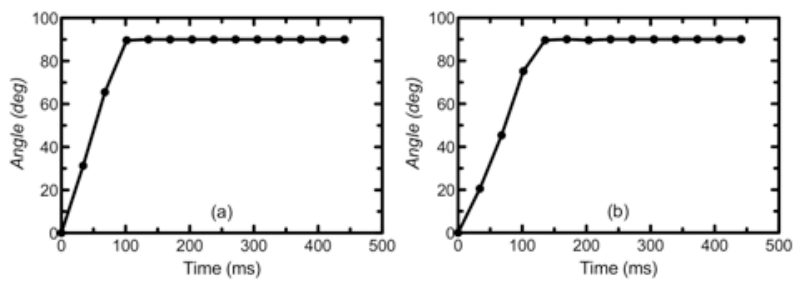

Fig. 2. Step response of the servomechanism for a reference value of $90^{\circ}$ with no load (a) and a load of $0.11 \mathrm{Nm}$ in (b).

Dynamic Motion
All motion profiles executed during the characterization of the ability of the servomechanism to replicate motion were successfully executed; these profiles contained velocities up to $20.6 \pm 4$ rad. $\mathrm{s}^{-1}$. Sections from representative manual motion profiles recorded with a load of $0.11 \mathrm{Nm}$ and with no load are shown in Fig. 3. The time delay in replicating the motion of the master encoder was $41 \pm 21 \mathrm{~ms}$, under maximum loading conditions.
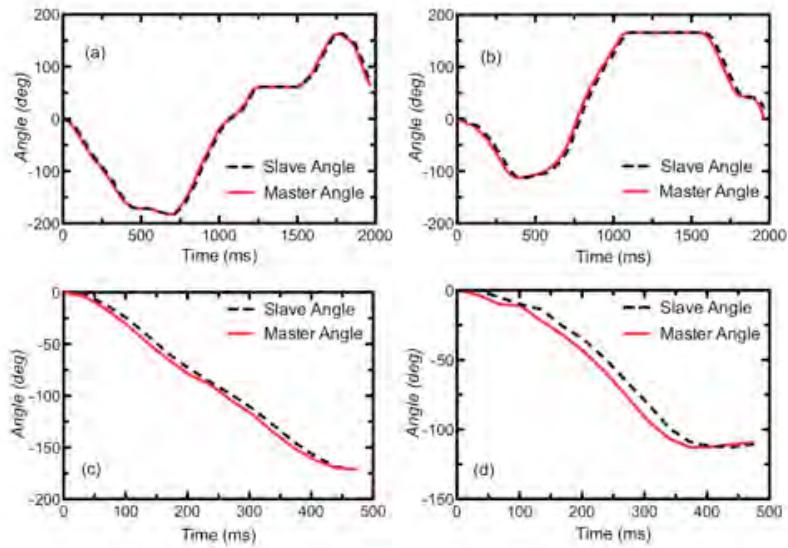

Fig. 3. Manual motion profiles (angular position) of the master and slave are plotted as a function of time: a) with no load b) with a load of $0.11 \mathrm{Nm} \mathrm{c}$ ) and d) are zoomed-in versions of the first $500 \mathrm{~ms}$ of profiles in a) and b), respectively, plotted to demonstrate the small delay in response.

\section{B. Evaluation of the MR-RCNS}

\section{Axial and Radial Motion Accuracy}

When replicating motion within the MRI scanner, an absolute error of $1.1 \pm 0.8 \mathrm{~mm}$ was measured when replicating axial motion over $100 \mathrm{~mm}$. The radial motion accuracy tests showed an absolute value error of $2 \pm 2^{\circ}$ for radial catheter motion replication over $360^{\circ}$. The type of pulse sequence, including the real-time acquisition, did not affect the axial nor radial accuracy of the system.

\section{Evaluation of the effects of the RCNS on MR images}

As expected, the SNR evaluation results showed that the worst-case SNR drop occurred when the RCNS was connected and the motors were moving (state 3 ). The SNR at this state was $130.6 \mathrm{~dB}$ for the SE images, which represented a drop of $2.5 \%$ from the baseline SNR.

\section{DISCUSSION}

We have presented an MR compatible master-slave catheter manipulator that captures the interventionalist's conventional motion on an input catheter and replicates that motion on a catheter within the bore of an MRI scanner. This MR-RCNS enables MR guided catheterization in a conventional MRI suite, removing the need to modify the conventional MRI facilities to accommodate in-suite image monitors and removing the ergonomic burden on the interventionalist. During operation within the MRI environment, the masterslave system showed to have an error of $1.1 \pm 0.8 \mathrm{~mm}$ when replicating axial motions over $100 \mathrm{~mm}$ and an error of $2 \pm 2^{\circ}$ 
for radial motion over $360^{\circ}$. The measurement of the radial motion replication accuracy is dependent on the catheter's flexibility - a more rigid catheter would be less prone to twisting and would provide more accurate replication readings. Overall, the errors in replication of the motion are acceptable for the majority of catheterization procedures especially taking into account the fact that position verification and catheter tip guidance is commonly performed using visual feedback. The interventionalist is simply using the master to drive the slave catheter and small errors in position do not represent a problem, as has been demonstrated in the previous non-MRI compatible RCNS implementation. Nonetheless, further reduction of the errors in motion replication can be achieved by either eliminating sources of slippage in the catheter manipulation (e.g. between the rollers and the catheter) or by utilizing direct feedback of the actual catheter position.

The time delay of motion replication using the RCNS was $41 \pm 21 \mathrm{~ms}$ which is significantly smaller than the earlier non MR-compatible version which had a delay of approximate $300 \mathrm{~ms}$ [8]. The shortening of the time delay is attributed to the use of an embedded system with a real-time control capability and an independent controller for each joint. The kinematics of the motions applied on the input catheter in our evaluations were very similar to the previous study [21]. Given the success in using the previous version of the RCNS (non-MRI compatible) during in-vivo cardiac ablation studies, we anticipate that the MR-RCNS described here will be at least equally successful as the delay in motion replication is smaller and therefore the new system is better capable of replicating dynamic motion[25].

An important aspect of introducing any mechanically driven system within an MRI suite is to ensure that the operation of such a device does not introduce undesirable RF noise in the MRI images. The presented results demonstrate a very small decrease in SNR during SE image acquisition, but no artifacts were observed. It must be noted that the type of MRIcompatible catheter used will dominate any local image artifacts and therefore local analysis was not covered in this study. Overall, the use of the MR-RCNS did not adversely affect the image quality and can be used during interventional procedures.

Our implementation does not include haptics feedback for the interventionalist. Although force information from the tip of the catheter interacting with the tissue would be beneficial in catheterization procedures, in conventional catheterization, the flexible body of the catheter as well as the viscous frictions of catheter-introducer/vessel prevents the interventionalist to obtain meaningful force feedback from the tip of the catheter. Therefore, in our implementation we have not pursued obtaining force feedback or developing a haptics interface. We believe any force feedback must be obtained from the tip of the catheter directly to be meaningful and this objective must be considered as part of a catheter design problem.

Further updates to this system require the miniaturization of the manipulator, which will enable the manipulation of multiple catheters and sheaths required during many catheterization procedures. The present design is compatible both with MRI and x-ray guidance and will represent an ideal solution for interventional suites that combine $\mathrm{x}$-ray and MRI guidance (XMR). Further studies, evaluating the performance of the MR-RCNS during real-time MRI guidance of procedures in vivo are also required.

\section{CONCLUSION}

We have introduced a magnetic-resonance-compatible remote catheter navigation system that observes the interventionalist's conventional motion on an input catheter in a master setup and replicates that motion through an MR compatible slave manipulator on a patient catheter. This system facilitates MRI-guided catheterization in conventional MRI scanners without the requirement of modifying the conventional MRI suite. The presented system also frees the interventionalist from the requirement to work within the constraining physical workspace of an MRI scanner.

\section{ACKNOWLEDGMENT}

The authors thank Maged Goubran and Eli Gibson for helpful assistance. M.T. acknowledges the support of the NSERC Collaborative Research and Training Experience (CREATE) Program in Computer-Assisted Medical Interventions (CAMI) at the University of Western Ontario. M.D. is a Career Investigator of the Heart and Stroke Foundation of Ontario.

\section{REFERENCES}

[1] C. Brasselet, T. Blanpain, S. Tassan-Mangina, A. Deschildre, S. Duval, F. Vitry, N. Gaillot-Petit, J. P. Clement, and D. Metz, "Comparison of operator radiation exposure with optimized radiation protection devices during coronary angiograms and ad hoc percutaneous coronary interventions by radial and femoral routes," Eur Heart J, vol. 29, pp. 63-70, Jan 2008.

[2] H. W. Lange and H. von Boetticher, "Randomized comparison of operator radiation exposure during coronary angiography and intervention by radial or femoral approach," Catheter Cardiovasc Interv, vol. 67, pp. 12-6, Jan 2006.

[3] A. M. Ross, J. Segal, D. Borenstein, E. Jenkins, and S. Cho, "Prevalence of spinal disc disease among interventional cardiologists," Am J Cardiol, vol. 79, pp. 68-70, Jan 11997.

[4] V. L. Roger, A. S. Go, D. M. Lloyd-Jones, R. J. Adams, J. D. Berry, T. M. Brown, M. R. Carnethon, S. Dai, G. de Simone, E. S. Ford, C. S. Fox, H. J. Fullerton, C. Gillespie, K. J. Greenlund, S. M. Hailpern, J. A. Heit, P. M. Ho, V. J. Howard, B. M. Kissela, S. J. Kittner, D. T. Lackland, J. H. Lichtman, L. D. Lisabeth, D. M. Makuc, G. M. Marcus, A. Marelli, D. B. Matchar, M. M. McDermott, J. B. Meigs, C. S. Moy, D. Mozaffarian, M. E. Mussolino, G. Nichol, N. P. Paynter, W. D. Rosamond, P. D. Sorlie, R. S. Stafford, T. N. Turan, M. B. Turner, N. D. Wong, and J. Wylie-Rosett, "Heart disease and stroke statistics--2011 update: a report from the American Heart Association," Circulation, vol. 123, pp. e18-e209, Feb 12011.

[5] J. Jayender, R. V. Patel, and S. Nikumb, "Robot-assisted Active Catheter Insertion: Algorithms and Experiments," International Journal of Robotics Research, vol. 28, pp. 1101-1117, Sep 2009.

[6] A. Al-Ahmad, J. D. Grossman, and P. J. Wang, "Early experience with a computerized robotically controlled catheter system," $J$ Interv Card Electrophysiol, vol. 12, pp. 199-202, Apr 2005.

[7] L. Cercenelli, E. Marcelli, and G. Plicchi, "Initial experience with a telerobotic system to remotely navigate and automatically reposition standard steerable EP catheters," ASAIO J, vol. 53, pp. 523-9, Sep-Oct 2007.

[8] Y. Thakur, J. S. Bax, D. W. Holdsworth, and a. M. Drangova, "Design and performance evaluation of a remote catheter 
Engineering, vol. 56, pp. 1901-1908, 2009.

[9] R. Beyar, L. Gruberg, D. Deleanu, A. Roguin, Y. Almagor, S. Cohen, G. Kumar, and T. Wenderow, "Remote-control percutaneous coronary interventions: concept, validation, and firstin-humans pilot clinical trial," J Am Coll Cardiol, vol. 47, pp. 296300, Jan 172006.

[10] S. Ernst, F. Ouyang, C. Linder, K. Hertting, F. Stahl, J. Chun, H. Hachiya, D. Bansch, M. Antz, and K. H. Kuck, "Initial experience with remote catheter ablation using a novel magnetic navigation system: magnetic remote catheter ablation," Circulation, vol. 109, pp. 1472-5, Mar 302004.

[11] M. N. Faddis, W. Blume, J. Finney, A. Hall, J. Rauch, J. Sell, K. T. Bae, M. Talcott, and B. Lindsay, "Novel, magnetically guided catheter for endocardial mapping and radiofrequency catheter ablation," Circulation, vol. 106, pp. 2980-5, Dec 32002.

[12] S. Pintillie, L. Biswas, S. Oduneye, K. Anderson, G. Wright, and P. Radau, "Visualization platform for real-time MRI-guided cardiac interventions," in 19th Scientific Meeting, International Society for Magnetic Resonance Imaging, Montreal, 2011.

[13] K. S. Nayak, C. H. Cunningham, J. M. Santos, and J. M. Pauly, "Real-time cardiac MRI at 3 tesla," Magnetic Resonance in Medicine, vol. 51, pp. 655-660, Apr 2004.

[14] S. Zhang, M. Uecker, D. Voit, K. D. Merboldt, and J. Frahm, "Real-time cardiovascular magnetic resonance at high temporal resolution: radial FLASH with nonlinear inverse reconstruction," $J$ Cardiovasc Magn Reson, vol. 12, p. 39, 2010.

[15] M. Uecker, S. Zhang, D. Voit, A. Karaus, K. D. Merboldt, and J. Frahm, "Real-time MRI at a resolution of $20 \mathrm{~ms}$," $\mathrm{Nmr}$ in Biomedicine, vol. 23, pp. 986-994, Oct 2010.

[16] B. A. Hoffmann, A. Koops, T. Rostock, K. Mullerleile, D. Steven, R. Karst, M. U. Steinke, I. Drewitz, G. Lund, S. Koops, G. Adam, and S. Willems, "Interactive real-time mapping and catheter ablation of the cavotricuspid isthmus guided by magnetic resonance imaging in a porcine model," Eur Heart J, vol. 31, pp. 450-6, Feb 2009.

[17] A. C. Lardo, H. Halpering, C. Yeung, P. Jumrussirikul, E. Atalar, and E. McVeigh, "Magnetic Resonance Guided Radiofrequency Ablation: Creation and Visualization of Cardiac Lesions," in MICCAI, 1998, pp. 189-196.

[18] R. Razavi, D. L. Hill, S. F. Keevil, M. E. Miquel, V. Muthurangu, S. Hedge, K. Rhode, M. Barnett, J. V. Vals, D. J. Hawkes, and E. Baker, "Cardiac catheterization guided by MRI in children and adults congenital heart disease," The Lancet, vol. 362, pp. 18771882, 2003.

[19] K. Ratnayaka and R. J. Lederman, "Interventional cardiovascular MR-The next stage in pediatric cardiology," Progress in Pediatric Cardiology, vol. 28, pp. 59-67, 14 DEC 2009.

[20] Y. Thakur, J. H. Cakiroglu, D. W. Holdsworth, and M. Drangova, "A device for real-time measurement of catheter-motion and input to a catheter navigation system," in SPIE, San Diego, CA, 2007.

[21] Y. Thakur, D. W. Holdsworth, and M. Drangova, "Characterization of catheter dynamics during percutaneous transluminal catheter procedures," IEEE Transactions on Biomedical Engineering, vol. 56, pp. 2140-2143, 2009.

[22] H. Elhawary, A. Zivanovic, B. Davies, and M. Lamperth, "A review of magnetic resonance imaging compatible manipulators in surgery," Proc Inst Mech Eng H, vol. 220, pp. 413-24, Apr 2006.

[23] N. E. M. A, "Determination of Signal-to-Noise Ratio (SNR) in Diagnostic Magnetic Resonance Imaging," NEMA Standard Publication MS 1- 2008.

[24] D. A. C. Kelley, "Measuring the effect of field strength on noise amplification factor," Concepts in Magnetic Resonance Part BMagnetic Resonance Engineering, vol. 31B, pp. 51-59, Feb 2007.

[25] Y. Thakur, D. L. Jones, A. Skanes, R. Yee, and M. Drangova, "Right-side RF ablation using remote catheter navigation: experimental results in vivo," J Cardiovasc Electrophysiol, vol. 23, pp. 81-7, Jan.

Mohammad Ali Tavallaei received his B.Sc. in electrical-electronics engineering from Urmia University, Iran and his M.Sc. in electrical-control engineering from Tabriz University, Iran in 2005 and 2008, respectively.
$\mathrm{He}$ is currently working towards his Ph.D. degree in biomedical engineering at the Graduate Program in Biomedical Engineering at the University of Western Ontario. He is also a member of the NSERC CREATE training program for Computer Aided Medical Interventions (CAMI) at the University of Western Ontario.

Yogesh Thakur received his B.A.Sc in electrical engineering, with a specialization in communications engineering and engineering management from The University of Ottawa in 2004 . In 2009 he completed his PhD in Biomedical Engineering from the University of Western Ontario.

Currently, he is a medical physicist with the Vancouver Coastal Health Authority and a clinical instructor in the Faculty of Medicine, University of British Columbia.

Syed Haider Syed Haider received a B.E. degree in electrical engineering from NED University, Karachi, Pakistan in 2000, a MS in electrical engineering from University of Texas at Arlington, USA in 2003 and M.Sc. in Biomedical Physics from Ryerson University, Toronto, Canada in 2008.

He joined the Robarts Research Institute in 2008 and worked on the design and construction of electro-mechanical prototypes for MR compatibility. Currently he is working as a research engineer in the focused ultrasound group at Sunnybrook Hospital, Toronto, Canada.

Maria Drangova received a B.A. Sc. Degree in engineering science from the University of Toronto, Toronto, ON, Canada, in 1984, and a Ph.D. degree in medical biophysics from the University of Western Ontario, London, ON, in 1993. She was a Postdoctoral Fellow at Stanford University. In 1997 she became a Scientist at the Imaging Research Laboratories, Robarts Research Institute, The University of Western Ontario, where she is currently a Professor in the Departments of Medical Biophysics and Medical imaging, as well as the program in Biomedical Engineering. She is also a Career Scientist with the Heart and Stroke Foundation of Ontario, Ottawa, ON. Her current research interests include developing novel cardiac imaging techniques for diagnosis and the guidance of interventional procedures. 